\title{
Über einige österreichische, besonders Tiroler Weiden.
}

Von Ad. Toepffer (München).

Auf Reisen in Tirol und Salzburg in den Sommern 1905 bis 1907 gemachte Beobachtungen an Weiden und Weidengallen, sowie die sich daran knüpfenden Studien haben mehreres für die Salicologie Beachtenswerte ergeben, das zum Teil schon in den Schedis zu meinem Salicetum exsiccatum (Salic.) verwertet, hier im Zusammenhange gegeben werden soll; der Übersichtlichkeit wegen wurden die Arten und Hybriden alphabetisch angeordnet; die mehrfach wiederkehrende Seiser Alp (Südtirol) ist durch S. A. gekürzt, die Staubblütenpflanze, resp. fruchttragende durch die üblichen Zeichen $\delta$, resp. $q$ bezeichnet, Salix stets durch $S$.; Höhenangaben annähernd.

S. appendiculata Vill. f. parva, , , S. A., zwisehen Felsen im Ochsenbachtal, ca. $2000 \mathrm{~m}$, Dolomit.

Nur $10-15 \mathrm{~cm}$ hohe, reich verzweigte Sträuchlein, mit Blättern von der Form typischer S. appendiculata (grandifolia Ser.), aber nur 20:12 mm, die größten derSommertriebe $30: 15 \mathrm{~mm}$, kahl oder mit verstreuten Härchen, nur die untersten und die jungen Triebe fein seidig; o Kätzchen $25 \mathrm{~mm}$ lang, $7 \mathrm{~mm}$ dick, kurz gestielt, von zwei laubblattähnlichen $(7: 5 \mathrm{~mm})$ Blättchen gestützt. - Gleiche Formen sah ich aus der Schweiz im Herbar des Herrn Dr. Hegi.

S. appendiculata Vill. f. proleptica ․ Tirol, St. Jodok, VIII. 1905 .

S. arbuscula L. f. androgyna S. A., Grünser Bühel, ca. 1900 m, Dol omit.

Vereinzelt Früchte unter normalen $\sigma^{\pi}$ Blüten. Übergangsbildungen nicht vorhanden.

S. arbuscula L. X caesia Vill. $\%$ (S. Trefferi Huter in Treffer, Exsicc., 1884).

Mit Recht haben Camus I (Monographie des Saules de France, 1904) 303 gen. Bastard aufgeführt; R. Buser hatte in Grem li, Neue Nachträge VI (1887), 76, behauptet, daß die von Treffer im Rainthal bei Taufers gesammelten und Flora exsicc. Austro-Hung., n. 1449, ausgegebenen Exemplare $S$. caesia var. angustifolia seien; die gleiche Angabe findet sich bei Wohlfahrt in Kochs Synopsis, Ed. 3, 2368, mit dem Zusatz: „Diese Form hat fast purpurea-artigen Habitus durch ihre verlängerten Blätter.

Daß einem so scharfen Beobachter wie Buser die Unterschiede des Bastardes von $S$. caesia entgangen sein sollten, ist 
kaum anzunehmen; vielmehr scheinen die von Treffer ausgegebenen Exemplare von verschiedenen Sträuchern zu stammen, denn meine Exemplare aus Baenitz, Herb. Europ., sind in der Tat schmalblättrige $S$. caesia; dagegen lag mir aus dem Herbar von Frau Andrée-Eysn die Nr. 1449 gen. Exsikkatenwerkes vor, die richtig hybrid ist; die nach dem Grunde keilig verlaufenden blaugrünen kahlen Blätter von derber Substanz sind in der vorderen Hälfte drüsig gesägt (bei $S$. caesia vollkommen ganzrandig); die Stützblätter des Kätzchens sind am Grunde seidig (bei $S$. caesia kahl); Kätzchenspindel wollig (bei S. caesia weichhaarig); Schuppen spärlich behaart, flach (bei $S$. caesia kahl, hohl), gleichfarbig oder oben dunkler (bei $S$. caesia alle oben dunkler); die Kapsel ist spitzer und etwas länger gestielt, die Drüse kräftiger und läng er als bei $S$. caesia, der Griffel dünner und die Narben tief geteilt (bei $S$. caesia ganz). - Da der obere Blattrand stark drüsig gesïgt ist, sehe ich als den einen parens typische $S$. arbuscula an, nicht Waldsteiniana, wie Huter in Österr. botan. Zeitschr., 1907, p. 436 , meint.

Die gleiche Form dieses Bastardes fand gen. Dame auch „im Suldenthal auf Bachschutt, ca. $2000 \mathrm{~m}$, Aug. 1879".

S. arbuscula L. X reticulata L. O f. medians Enander in sched. ad Enand., Salic. Scandin. exsicc., 1905; Salic., n. 106 (1908), icon. photogr.

S. Ganderi Huter sec. Gandoger in Flora, XL (1882), 231.

S. A., Abhänge des Platten, ca. $2100 \mathrm{~m}$, Dolomit. 12. VII. 1905. - Salic. n. 106, icon photogr.

Habitus der $S$. reticulata, doch die kleinen Zweige aufstrebend; Äste kastanien- bis rotbraun, etwas glänzend; Knospenschuppen kahl, gelbbraun, bleibend; Blätter oval bis verkehrt-eiförmig (1:0.8-3:1.5 cm), auf $0.3-0.6 \mathrm{~cm}$ langen rinnigen, rötlichen Stielen, oberseits glänzend dunkelgrün, durch das vorspringende Adernetz (Rippe aber eingesenkt) etwas runzelig, unterseits glauk, jung lang seidig behaart, später kahl, die gelbgrüne Rippe und Nerven hervortretend, Netz nur dunkler gefärbt, nicht hervortretend; Rand umgebogen, entfernt drüsig gezähnt; Kätzchen auf $2-3 \mathrm{~cm}$ langen, roten, dünn weiß behaarten, mit $3-4$ den Laubblättern gleichenden Stützblättern versehenen Stielen, mit und ohne Knospen in den Blattachseln, 2-3 cm lang, $0.5-0.7 \mathrm{~cm}$ dick, walzig, oft gekrümmt, unten meist lockerblütig; Schuppen einfarbig, rundlich oval, ein Drittel so lang als der Fruchtknoten, innen und außen lang seidig behaart; Fruchtknoten 3-4 mm lang, sitzend, eiförmig, stumpf, dunkelrot, dicht weiß seidig behaart; Griffel rot, halb oder bis zum Grunde gespalten, dünn, mit zweiteiligen, roten Narben; Drüse doppelt, äußere klein, innere breit, oben gekerbt, bis ein Viertel des Fruchtknotens aufragend.

Die Tiroler Pflanze gleicht der von Enander l. c. photographisch gegebenen Form n. $10^{1} / 2 a$ : sie weicht ab durch we- 
niger stark gezähnte Blätter, kräftigere Kätzchen, sitzende Kapsel, zierlicheren Griffel und geteilte Narben.

S. caesia Vill. Typische S. caesia Vill. ähnelt außerordentlich S. myrtilloides L., mit der sie von vielen Autoren verwechselt wurde; aber neben dieser elliptischen Form variieren die Blätter einerseits bis schmal-lanzettlich, anderseits bis fast kreisrund; sie sind stets ganzrandig und bei uns immer kahl; nur Turezaninow (Flora baicalensi-dahurica, 394) erwähnt eine Form aus Sibirien mit jung unterseits lang behaarten Blättern.

Von Blattformen erwähnt Seringe (Essai Saules Suisse, 1875,27 ) eine var. $B$. macrophylla, die er jedoch nur kultiviert kennt; und obwohl die leicht zu kultivierende Art sehr konstant bleibt, kommen doch einzelne Stöcke vor, die (wie auch S. myrti7loides L.) wesentlich größere Blätter bilden und zu bis mannshohen Sträuchern heranwachsen; die $\&$ Pflanze geht auch in den Gärten als $S$. caesia, die $\delta^{\top}$ als $S$. Zabeli oder auf Hochstämme veredelt und als Trauerbäumchen gezogen, als S. Zabeli pendula Hort.; mit ihren schlanken, blütenbeladenen Zweigen, aber auch später mit ihrem dunkelgrünen Laub ist sie eine unserer schönsten Zierweiden. Knospen.

$S$. Wimmeri Hartig unterscheidet sich nur durch behaarte

R. Buser (in Gremli, l. c., 76) nennt eine var. angustifolia, ebenso Camus, I., 143, als var. angustifolia Mutel, Fl. Dauph. ed. 2, 563; ihre Abbildung tab. 10, fig. $A$, zeigt Blätter von 15-18: $4 \mathrm{~mm}$, was mit den von Treffer angegebenen unter $S$. arbuscula $\times$ caesia erwähnten Exemplaren ubereinstimmt.

Die Abbildungen der Kapsel bei Reichenbach und Camus stimmen weder miteinander noch mit meinen Beobachtungen überein; ich fand sie stumpfeiförmig (fast wie bei $S$. purpurea), mit kurzem, dickem Griffel und roten ungeteilten Narben; Schuppe rundlich, hohl, kahl, halb so lang als die Kapsel und diese am Grunde halb umfassend; Drüse unten dicklich, nach oben verschmälert, die Basis der sehr kurz gestielten Kapsel kaum erreichend.

In dem von Herrn Ingenieur A. A r tzt bearbeiteten botanischen Teil von Prosliner, Das Bad Ratzes (1895), ist auf Grund einer Mitteilung des $\dagger$ Herrn Generalsuperintendent Bertram in

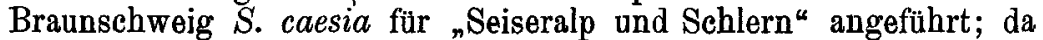
ich auf mehr als 50 Exkursionen in gen. Gebiet von dieser Spezies nichts entdecken konnte, bemühte ich mich um Einsichtnahme des B ertr a m schen Herbars, in dem aber weder im Braunschweigischen, noch im Wolfenbütteler Teil unsere Art von S. A. und Schlern enthalten ist; wohl aber fand ich $S$. arbuscula mit ovalen, sehr schwach gezähnten, blaugrünen Blättern als fast kriechende Sträucher an quelligen Stellen, die die größte Ähnlichkeit mit $S$. caesia Vill. oder auch myrtilloides L. zeigten; da gleiche Exemplare im Herbar 
Bertram vorliegen, habe ich Grund zu der Annahme, daß der gelehrte Herr Verfasser der "Flora von Braunschweig“" diese ursprünglich für $S$. caesia Vill. gehalten, später auch seinen Irrtum eingesehen, aber nicht mehr Gelegenheit gehabt hat, die an A rtzt gegebene Notiz zu berichtigen; $S$. caesia Vill. dürfte für "Seiser Alp und Schlern" zu streichen sein. - Ein"Synonym für $S$. caesia Vill. ist S. myrtilloides Willdenow; S. myrtilloides Villars ist wiederum S. arbuscula L.; man sieht, wie die älteren Autoren die ähnlichen Spezies zusammenwarfen. - In Pa cher (Systematische Aufzählung der in Kärnten wildwachsenden Gefäßpflanzen, 1884), 19, wird S. myrtilloides L. „Im Möllthal Blmfld. u. Schwab“ aufgeführt; im Herbar Pacher, das ich dank der Liebenswürdigkeit der Verwaltung des Naturhistorischen Landes-Museums von Kärnten durchsehen konnte, ist diese Art nicht enthalten; da nach der sonstigen geographischen Verbreitung ihr Vorkommen südlich der Alpen nicht zu erwarten ist, glaube ich nach Obgesagtem annehmen zu müssen, daß auch hier eine Verwechslung der $S$. arbuscula L. $\beta$. humitis 2 brevifolia Anderss. in DC., Prodrom., XVI, 2., 248 (= myrtilloides Vill.) mit S. myrtilloides L. vorliegt.

S. crataegifolia Huter in sched. et in Österr. botan. Zeitschr., 1907, p. 471 [non Bert.]. Die Originale der S. crataegifolia Bert., die ich im Herb. Mus. Paris. sah, weichen von den von Huter ausgegebenen wesentlich ab; die Blätter der Bertolinischen Pflanze sind oval, stumpf, $80: 43 \mathrm{~mm}$, die der Huter schen eilanzettlich, spitz, $50: 17 \mathrm{~mm}$; sollte sich, was ich zurzeit nicht teststellen kann, eine Identität der Kätzchen ergeben, so müßte die Hutersche Pflanze als var. angustifolia bezeichnet werden.

S. daphneola Huter in Österr. botan. Zeitschr., 1907, p. 470 [non Tausch] $=$ arbuscula $\times$ hastata.

Herr Huter hat bei der Identifikation seiner Pflanze mit der Tauschschen rom Riesengebirge übersehen, daß in den Sudeten der eine parens $S$. arbuscula $L$. nicht vorkommt; ich halte mit allen Autoren die Pflanze von der "Pantsche-Wiese“ für eine kahle Zwergform von S. Lapponum L.

S. hastata L. zeigt meist rein grüne Kapseln, doch kommen auch rot überlaufene und grün punktierte, oder nur in der unteren Hälfte rote, an den gleichen Standorten vor.

$S$. $h$. f. pseudohermaphrodita: S. A., Wiese unter dem Grünser Bühel, $2000 \mathrm{~m}$. Der Strauch hat das Aussehen einer o Pflanze, doch sind wenige Staubblätter vollkommen entwickelt, die meisten zeigen bereits Übergänge zum $\&$ Geschlecht; in einem Ährchen fand sich eine einzelne Kapsel ausgebildet, ein Kätzchen zeigte etwa zur Hälfte $\sigma^{\star}$ und $q$ Bläten und zwei Kätzchen des Strauches waren rein o.

S. herbacea L. (Vgl. Österr. botan. Zeitschr., 1904, Nr. 5.) - Die Farbe der Antheren ist vor der Blüte lebhaft purpurn, dann goldgelb. 
Früher hatte ich die $\&$ Blüten der Art stets zweidrüsig gefunden (auch $\mathrm{H}_{\mathrm{offm}} \mathrm{nn}$, Historia Salicum, Fasc. IV [1787], t. XX, und Hartig, System und Beschreibung der europäischen Weiden [1850], tab. 105 [35b], zeichnen sie zweidrüsig), indessen habe ich nunmehr in der Gastein (Hüttkogel und Tisch [Salie., n. 27 u. 28]) auf Glimmerschiefer und auf der Seiser Alp auf Melaphyr Eixemplare gesehen, deren meiste Blüten eindrüsig waren; auch Enande r (Salices Scand. exs. u. 21) kennt \& Blüten mit einem inneren Nektar; bei den von ihm angegebenen Modifikationen der Blüten mit zwei Drüsen ist die äußere stets heil, die innere ganz oder zwei- oder dreilappig.

Zu den von mir l. c. genannten Varietäten, resp. Formen treten noch var. ovalis Norman (Florae areticae Norvegiae species nonnullae novae vel minus cognitae in Christiania Förhandl., 1893, 38): „Meiste Blätter oval, seltener elliptisch-oval, gewöhnlich nicht gestutzt, wenige verkehrt ei-kreisrund oder rundlich gestutzt" und

f. acutifolia (Salic., n. 70 A) S. A., nahe dem Balstein, ca. $2000 \mathrm{~m}$. Linn é bildet in Fl. Lappon. die o Pflanze mit spitzen Blättern ab; hier ist es die + Pflanze, die solch scharf zugespitzte Blätter führt; sie ist offenbar aus einem Stock gewachsen, bedeckt eine ca. $4 \mathrm{~m}^{2}$ große Fläche in dichtestem Rasen, der alle andere Vegetation verdrängt hat; trotz sorgfältigsten Suchens konnten nur drei \& Kätzchen entdeckt werden, deren Blüten eindrüsig waren.

Wohlfahrt (Koch, Syn. etc.), 2308, sagt "sehr selten androgyn"; da mir sonst aus der Literatur nichts über diese Form bekannt geworden, muß ich annehmen, daß hier eine Verwechslung mit Bertolonis f. monoica (vidi lusum monoicum, a mentis $\sigma^{7}$ et $q$ in eodem individuo, Bert., Fl. Ital. X., 307) vorliegt.

$S$. myrsinites L. Auf der S. A. soll auch die var. Jacquiniana vorkommen; ich fand bisher nur var. serrata (Salic., n. 72).

Partielle Phyllomanie. S. A. am Grünser Bühel fand ich eine Pflanze mit teilweise verlaubtem Kätzchen. Über den Stützblättern finden sich einige taube Früchte, dann wechseln vergrünte Blüten mit normalen fertilen $a b$; die untersten zeigen verlaubte und verdickte Deckschuppe mit drüsigem Rande; das Nektarium ist erhalten, die Karpelle haben sich zu einem normalen und einem verkümmerten Laubblatt ausgebildet, deren untere Ränder noch zusammenhängen und eine Sproßknospe umschließen; die mittleren vergrünten Fruchtkuoten zeigen noch deutlicher ihre Entstehung aus den Karpellen; hier sind Deckschuppe und Drüse normal erhalten, die verlaubten Kapselklappen aber an der inneren Seite ganz, an der äußeren zum Teil kapuzenförmig zusammengewachsen; Griffel und Narbe fehlen; an der Spitze des Kätzchens finden sich Phyllome, in denen auch Drüse und Deckschuppe zu kleinen Laubblättern umgewandelt sind; von den Karpellen aber, die nur noch am Grunde zusammenhängen, ist eines zu einem rundlichen, das andere zu einem auch in der Größe vollkommen normalen Laub- 
blatt ausgebildet, die sich von denen der Zweige nur durch stärkere Behaarung unterscheiden; in der Achse des verbildeten Fruchtknotens steht die nackte Knospe eines neuen Sprosses.

S. myrsinites var. serrata $\times$ retusa. - $\mathrm{Zu}$ sämtlichen auf S. A. gesammelten Exemplaren paßt die Beschreibung von S. retusoides J. Kerner (Verhandl. zool.-botan. Ges. Wien, XII [1862], 122), nur weichen die Blätter durch die ringsum drüsige Serratur und das Schwarzwerden beim Trocknen ab; sie stehen daher sämtlich der $S$. myrsinites näher; die Kätzchen gleichen denen der $S$. retusa var. Kitaibeliana, auch die Blüten in den gelben Deckschuppen und kahlen Kapseln; die Beteiligung von S. Myrsinites zeigt sich aber auch hier in den roten Adern der Deckschuppe und der Kerbung des Randes.

Bei Exemplaren rom Platten (Dolomit., ca. $2000 \mathrm{~m}, 12$. VII. 1905), die ich als f. super-myrsinites bezeichne, zeigt sich die nähere Verwandtschaft mit $S$. myrsinites in der wimperigen Behaarung des ringsum gesägten Blattrandes und dem Fehlen gestutzter oder ausgerandeter Blätter.

f. medians, Roßzähne, $2000 \mathrm{~m}$, Dolomit., 16. VII. 1905; Platten, ca. $2200 \mathrm{~m}$, 오 (Salic., n. 73) und Schlern am Touristensteig $\sigma^{7}$ in gleicher Höhe (Salic., n. 74), Juli 1907, zeigt nicht gewimperte, aber ringsum drüsig gesägte jüngere Blätter in der Form von $S$. myrsinites und gestutzte oder ausgerandete (ebenfalls ringsum drüsig gesägte) ältere Blätter wie $S$. retusa, beim Trocknen aber schwärzend.

Eine dritte Form der gleichen Verbindung sammelte ich $\delta^{\pi}$ am Grünser Bühel, ca. 2100 m, Dolomit., 15. VII. 1905, die ich als

S. myrsinites $\times$ retusa var. serpyllifolia bezeichnen muß; eine Photographie habe ich Salic., n. 132, gegeben; sie stimmt mit $S$. semiretusa G. Beck in der Behaarung der jungen Äste und Blätter, den $10-18$ blütigen Kätzchen mit 4-5 mm langen Staubblättern und gelbgrünen, fast kahlen Tragschuppen überein, weicht aber durch 10-15:3-5 mm (bei semiretusa 10-15:4-7) nach beiden Enden fast gleichmäßig verschmälerte, ringsum entfernt scharf drüsig gesägte Blätter ab. - Da die Blätter auffallend klein sind und gestutzte fehlen, so ist als der eine parens S. retusa var. serpyllifolia mit Sicherheit anzunehmen.

S. nigricans Sm. f. metamorpha, Tirol austral., mittleres Duronthal, 26. VII. 1905.

Die schon reifen 은 Kätzchen zeigen neben gut ausgebildeten Kapseln solche mit Umbildungen der Karpelle in Staubblätter, ohne daß diese Metamorphose vollendet ist; sämtliche Blüten sind rückwärts gerichtet. - Salic., n. 126, habe ich Umbildungen $\sigma^{7}$ Blütenorgane in $O$ als vielleicht durch die abnorme Witterung des Frühjahrs bedingt angegeben; auch für diese $S$. nigricans möchte ich das Gleiche voraussetzen, zumal auch das Laub merkwürdige Ver- 
schiedenheiten aufweist; die Langtriebe der $2 \mathrm{~m}$ hohen Sträucher tragen Blätter von verkehrt-eiförmiger Gestalt, mit langer Spitze, die mittleren $40: 22 \mathrm{~mm}$; die der mittelhohen Zweige sind mehr lanzettlich gestaltet, im Mittel $35: 10 \mathrm{~mm}$, die der untersten endlich schmallanzettlich, $15: 4 \mathrm{~mm}$; ich vermute, daß der Strauch, auch nach dem zurückgebliebenen Unrat zu schließen, während der Schneeschmelze längere Zeit im Wasser stand und dies eisige Wasser die Verschiedenheit der Blätter sowie die Umbildung der Blüten verursachte.

S. petandra L. var. lanceolata S. A. Geröll im Saltariabach oberhalb der Postsenne, ca. $1850 \mathrm{~m}$, sehr zahlreich $\sigma^{\circ}$ und $q$ in 1-2 $\mathrm{m}$ hohen Sträuchern; die Drüsen des Blattrandes waren außerordentlich harzreich und der Duft dieses Harzes, fast wie Perubalsam, erfüllte die Luft auf weite Strecken. - Salic., n. 75. $q$.

Ob die Synonyme 1. lanceolata Anderss., Sal. Lap. (1845), 13, und die var. angustifolia Anderss., Meyer, Camus zu unserer Pflanze gehören, vermag ich nicht zu sagen; jedenfalls sind mir aus der Ebene und aus dem Norden derartige Exemplare nicht zu Gesicht gekommen, dagegen mehrfach aus Tirol und der Schweiz; ich halte diese Form daher für alpin.

S. reticulata L. (Vgl. Österr. botan. Zeitschr., 1904, Nr. 5.) - Unter den l. c. angeführten Varietäten fehlt

var. angustifolia Borzi (Compend. della flora forest. ital. [1885], 137). Vergleicht man des Autors Worte „foglie bislunghe obovale" mit der Beschreibung var. cuneata Bornmüller (Mitt. Thür. Bot. Ver. VIII [1895], 39, „Blätter nach der Basis keilförmig verlaufend, doppelt-, einzeln dreimal so lang ale breit" ${ }^{*}$, so ist kein Zweifel über die Identität beider Formen, nur hat der Borzi sche die Priorität, während der Born müllers bezeichnender ist; übrigens sind derartige Formen schon fruher beobachtet: Ledebour, Flora Rossica, III (1853), 623, sagt: „Specimen unicum foliis obovati-oblongis, basi cuneatis, in terra Tschutschkorum ad sinum St. Laurentii lectum possideo."

Großblättrige Formen, die hierzu gehören, sah ich aus Norwegen, Lille Elvedal, von Haglund und Källström gesammelt; mit etwas kleineren Blättern sammelte ich sie \& S. A., Roßzähne, $2100 \mathrm{~m}, \delta^{\star}$ Südtirol, am Tschagerjoch, ca. $2000 \mathrm{~m}$, ㅇ Zentraltirol, Wildlahner Thal am Übergang zur Geraer Hütte; die Blattgröße schwankt zwischen 19-10 mm Länge bei 8-4 mm größter Breite; nicht alle Blätter sind gleichmäßig keilförmig, einzelne sind rundlich, die übrigen zeigen Zwischenformen.

Die Abbildung bei Schröter, Pflanzenleben der Alpen (1905), 208, Fig. 76, n. 6, „besonders schmales und langes Blatt (33: $17 \mathrm{~mm})$ stellt ein Mittelding dar zwischen der gen. var. angustifolia Borzi und einer S. A. am Grünser Bühel und später auch an den Roßzähnen gesammelten Form, auf die der Name angustifolia noch besser passen würde; das Verhältnis der Länge 
zur Breite bei dieser ist stets über $2: 1$ bis fast $3: 1(18-35 \mathrm{zu}$ $7-12 \mathrm{~mm}$ ), die Blätter sind aber nicht keilig, sondern in der Mitte am breitesten und verschmälern sich gleichmäßig nach beiden Enden, so daß also auch der obere l'eil der Blätter spitzlich erscheint; besonders schön sah ich schmalblättrige Formen aus Nordamerika (Patterson, Colorado Flora, Nr. 135, Mount about Headwaters of Clear-Creek, 1885, im Hb. Mus. Paris.), das Verbältnis war hier bis $5: 1$, die $\delta$ und of Kätzchen sehr klein.

Man könnte nach den Kernerschen Worten bei seiner var. vestita (Form der Schieferalpen) verleitet werden zu der Annahme, in den Schieferalpen komme ausschließlich die Form vor. Das ist unrichtig; anch dort ist die verkahlende Form die typische und die behaarte äußerst selten. Mit dem Substrat hat, wenigstens was seine chemische Beschaffenheit betrifft, die var. sericea Gaudin (Flora Helvet. VI [1830], 256, - Kerner erst 1860) nichts zu tun; sie wächst auf dem Radstädter Tauern (Windfeld, leg. M. Eysn) auf Urgestein, auf dem Platten, S. A., $2300 \mathrm{~m}$ (Salic., n. $89 \%$ u. 90 ठ) auf Dolomit, an beiden Stellen im Verein mit typischer $\dot{S}$. reticulata, welche bei dieser Höhe naturgemäß kleinblättriger ist als in tieferen Lagen. Aber während die tief im Boden wurzelnden Pflanzen ihren Haarschutz zeitig abwerfen, können die in lockerem Geröll wachsenden Formen, deren Wurzeln durch das herabrieselnde Schmelzwasser vielfach entblößt sind, dieses Schutzes nicht entbehren und behalten ihr Haarkleid auch oberseits während des ganzen Sommers. Mit S. vestita Purch hat unsere $\nabla$ arietät nichts zu tun, ebensowenig wie var. villosa Ledeb. 1. c., welche nur unterseits mit langen Haaren bekleidet bleibt.

$S$. retusa L. f. metamorpha S. A., Nordabhänge der Roßzähne, Juli 1907 (Salic., n. 144). - Die \& Kätzchen weisen Umbildungen der Karpelle in $\delta$ Blütenorgane auf, ohne Vollendung der Metamorphose. - In der Literatur finde ich nur einmal androgyne Blüten an S. var. Kitaibeliana (Wimmer, Salices Europaeae [1866], 124), es scheinen Blütenmetamorphosen bei dieser Art selten zu sein.

Die nachstehende Liste der von mir beobachteten Weidengallen mag als kleiner Beitrag zu ibrer geographischen Verbreitung dienen. Die Nomenklatur ist nach Darboux et Houard, Catalogue systematique des Zoocécidies de l'Europe et du Bassin méditerranéen (1901). Ich mußs es dahingestellt sein lassen, ob die auf verschiedenen Pflanzen vorkommenden Gallbildungen gleichen Aussehens von gleichen oder verschiedenen Tieren herrühren.

Blattrandrollung durch Eriophyiden an:

S. glabra Scop., Schlern, $1800 \mathrm{~m}$; hastata L., S. A., Ochsenbachthal, $2000 \mathrm{~m} ; S$. incana Schrank, Frötschbachthal ober Ratzes, $1100 \mathrm{~m}$. 
Eriophyidengallen, cephaloneonartige, an:

S. hastata L., Mittleres Duronthal, ca. $1700 \mathrm{~m}$; S. reticulata L., S. A., Grünser Bühel, $2000 \mathrm{~m}$; S. retusa L., S. A., Mahlknecht, $2100 \mathrm{~m}$.

Nematus gallicola Steph. an

S. arbuscula L., S. A., Spitzbühel, $1900 \mathrm{~m}$; S. glabra Scop., S. A., Spitzbühel, $1900 \mathrm{~m}$; S. hastata L., Schlern, $2000 \mathrm{~m}$.

Nematus ischnocerus Thomas an

S. hastata L., S. A., $1900 \mathrm{~m}$; S. Mielichhoferi Kerner, S. A., $1900 \mathrm{~m}$ (Salic. n. 148); S. nigricans Sm., S. A., Ochsenbachtal, $1900 \mathrm{~m}$.

Nematus vesicator Bremi an

S. purpurea L., Südtirol, Eggenthal vielfach; Nordtirol, Schmirner Thal, zahlreich.

Nematus viminalis (err. L.) ( $N$. gallarum Hart) an

S. arbuscula L., Schlern, $1800-2000 \mathrm{~m}$, mehrfach; S. hastata L., Schlern, Platten, Mahlknecht (Salic., n. 98a), 1900 bis $2100 \mathrm{~m} ;$ S. purpurea L., zwischen Waidbruck und Kastelruth, 700-900 m, Eggenthal, Schmirner Thal, überall zahlreich; S. reticulata L.. S. A. und Schlern (Salic., n. 98b), 2000-2200 m; S. retusa L., S. A., Schlern, Platten, 1900-2200.

Oligotrophus capreae Winn. an

S. appendiculata Vill., Nordtirol, St. Jodok, ca. $1100 \mathrm{~m}$.

Perrisia terminalis H. Loew. an

S. hastata L., S. A., Goldknopf, $2200 \mathrm{~m}$.

Rabdophaga rosaria $\mathrm{H}$. Loew. an

S. caprea L., Laranzer Wald bei Kastelruth, ca. $800 \mathrm{~m}$.

Rabdophaga Salicis Schrank an

S. arbuscula L. (Salic., n. 52), S. A., 1800 m, Platten, $2000 \mathrm{~m}$ (die Exemplare entsprechen durchaus denen von Dittrich und Pax, Herbarium cecidiologicum, n. 260, an $S$. helvetica ausgegebenen, welche von Rhabd. dubia Kieff. herrühren sollen); S. purpurea L., Eggenthal bei Birchabruck, zahlreich.

Pontania Kriechbaumeri Konow an

S. incana Schrank., Kardaun bei Bozen, bei Ratzes, $1100 \mathrm{~m}$, St. Jodok, $1100 \mathrm{~m}$. (Es bleibt zu unt ersuchen, ob der Erzeuger mit Nematus bellus Zadd. identisch ist.)

Phytoptus sp. an

S. pentandra L. var. lanceolata (Salic., n. 149) gehört vielleicht zu den oben erwähnten cephaloneonartigen Eriophyidengallen. S. A., Saltariathal, bei der Postsenne, ca. $1800 \mathrm{~m}$. 